\title{
On Algebraic Equations Satisfied by Hypergeometric Correlators in WZW Models. I.
}

\author{
Boris Feigin $^{1}$, Vadim Schechtman, ${ }^{2, \star}$ and Alexander Varchenko ${ }^{3, \star}$ \\ 1 Landau Institute for Theoretical Physics, Moscow, Russia \\ 2 Dept. of Mathematics, SUNY at Stony Brook, Stony Brook, NY 11794, USA \\ 3 Dept. of Mathematics, University of North Carolina at Chapel Hill, Chapel Hill, NC 27599, USA
}

Received: 15 July 1993

\begin{abstract}
It is proven that integral expressions for conformal correlators in $\operatorname{sl}(2)$ WZW model found in [SV] satisfy certain natural algebraic equations. This implies that the above integrals really take their values in spaces of conformal blocks.
\end{abstract}

\section{Contents}

1. Introduction . . . . . . . . . . . . . . . . . . . . . 173

2. Spaces of Conformal Blocks . . . . . . . . . . . . . . . . . . . . . . . . . 174

2.1 Representations of $\mathfrak{g}$. . . . . . . . . . . . . . . . . . . . . . . . . . 175

2.2 Representations of $\widehat{\mathfrak{g}}$. . . . . . . . . . . . . . . . . . . . . . . . . . 175

2.3 Spaces of Coinvariants . . . . . . . . . . . . . . . . . . . . . . . . . 176

3. Sending to Differential Forms . . . . . . . . . . . . . . . . . . . . . . . . 179

3.1 Configurational Arrangements . . . . . . . . . . . . . . . . . . . . . . 179

3.2 Resonances at Infinity . . . . . . . . . . . . . . . . . . . . . . . . . 180

3.3 The Map to Differential Forms . . . . . . . . . . . . . . . . . . . . . . 181

3.4 Truncation . . . . . . . . . . . . . . . . . . . . . . . . . . . . . . . 182

3.5 Proof of Theorem 3.4 .1 . . . . . . . . . . . . . . . . . . . . . . . . . 182

References . . . . . . . . . . . . . . . . . . . . 184

\section{Introduction}

Let $\mathbb{P}^{1}$ be a complex projective line with a fixed coordinate $z, \mathbb{A}^{1}=\mathbb{P}^{1}-\{\infty\}$. Let $\mathfrak{g}$ be a complex simple Lie algebra with a fixed invariant scalar product $($, ) defining the symmetric invariant tensor $\Omega \in \mathfrak{g} \otimes \mathfrak{g}, L_{1}, \ldots, L_{n+1}$ its irreducible representations. Set

$$
W=\left(L_{1} \otimes L_{2} \otimes \cdots \otimes L_{n+1}\right)_{\mathfrak{g}} .
$$

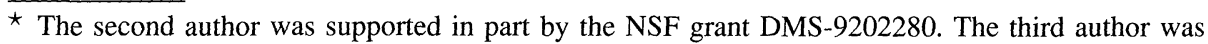
supported in part by the NSF grant DMS-9203939
} 
(For a representation $M$, we will denote be $M_{\mathfrak{g}}$ the space of coinvariants $M / \mathfrak{g} M$.) Consider the trivial vector bundle $\widetilde{\mathscr{W}}$ over the $n$-dimensional affine space $\mathbb{A}^{n}$ with the fiber $W$. The system of the Knizhnik-Zamolodchikov differential equations (cf. $[\mathrm{KZ}])$

$$
\frac{\partial \phi(z)}{\partial z_{i}}=-\frac{1}{\kappa} \sum_{j \neq i} \frac{\Omega_{\imath j} \phi(z)}{z_{i}-z_{j}}
$$

$i=1, \ldots, n$, defines the flat connection in $\widetilde{\mathscr{W}}$. Here $\phi(z)=\phi\left(z_{1}, \ldots, z_{n}\right) \in W, \Omega_{\imath \jmath}$ are the usual linear operators in $W$ defined using $\Omega, \kappa \neq 0$ is a complex parameter.

Suppose that $\kappa=k+g$, where $g$ is the dual Coxeter number of $\mathfrak{g}$, and $k$ is a positive integer. Let $\theta \in \mathfrak{g}$ be the highest root normalized in such a way that $(\theta, \theta)=2$. Suppose that highest weights ${ }_{i}$ of representations $L_{i}$ satisfy inequalities $\left({ }_{i}, \theta\right) \leq k$. In that case the flat bundle $\widetilde{\mathscr{W}}$ is not irreducible: it admits a certain remarkable quotient $\mathscr{W}$ defined by some set of algebraic equations.

Let us describe this quotient bundle explicitly for the case $\mathfrak{g}=s l(2)$. Pick Chevalley generators $e, f, h \in \mathfrak{g}$. Suppose that $L_{i}$ has the spin $m_{i} / 2$. Then the fiber of $\mathscr{W}$ at the point $\left(z_{1}, \ldots, z_{n}\right)$ is the quotient of $W$ over the subspace spanned by elements of the form

$$
\sum_{i=1}^{n}\left(z_{i} f^{(i)}\right)^{k-m_{n+1}+1} x
$$

for some $x$, where $f^{(i)}$ denotes the operator $f$ acting at the $i^{\text {th }}$ factor. For details, see Sect. 2 below. This quotient was described (in a slightly different form) in [TK].

For an arbitrary $\mathfrak{g}$ the definition is analogous, cf. [FSV]. The bundle $\mathscr{W}$ is the fundamental object of the Wess-Zumino-Witten model of the Conformal Field Theory (with the central charge $k$ ). We will call it the bundle of conformal blocks since its horizontal sections are called "conformal blocks" by physicists.

In the work [SV] we have constructed horizontal sections of the dual bundle $\widetilde{\mathscr{W}}^{*}$, i.e. solutions of $\mathrm{KZ}$ equations, using generalized hypergeometric integrals. The main result of the present paper (Theorem 3.4.1, Corollary 3.4.2) and of the next one says that the above integrals actually lie in the subbundle $\mathscr{W}^{*} \subset \widetilde{\mathscr{W}^{*}}$. In other words, the above solutions satisfy the algebraic equations dictated by the operator (2) (or its analogue for an arbitrary $\mathfrak{g}$ ). This result was announced in [FSV].

The present paper treats the case $\mathfrak{g}=\operatorname{sl}(2)$. We decided to devote a separate paper to this case since it is simpler in formulation and admits a proof which is simpler and different from the general case. The next paper will be devoted to the case of a general simple Lie algebra.

\section{Spaces of Conformal Blocks}

Throughout this paper $\mathfrak{g}$ will denote the complex Lie algebra $\operatorname{sl}(2) . \mathbb{N}$ will denote the set of nonnegative integers. 


\subsection{Representations of $\mathfrak{g}$}

Fix the Chevalley generators $e, f, g \in \mathfrak{g}$; put $\mathfrak{n}_{-}=\mathbb{C} f, \mathfrak{n}_{+}=\mathbb{C} e, \mathfrak{h}=\mathbb{C} h$, so that $\mathfrak{g}=\mathfrak{n}_{-} \oplus \mathfrak{h} \oplus \mathfrak{n}_{+}$is the Cartan decomposition. We fix an invariant form (, ) on $\mathfrak{g}$ normalized in such a way that $(h, h)=2$. If $M$ is a representation of $\mathfrak{h}$ we set $M_{m}=\{x \in M \mid h x=m x\}$.

For a complex number $m$ denote by $M(m)$ the Verma module over $\mathfrak{g}$ which is generated by a vector $v$ subject to the defining relations $e v=0$ and $h v=m v$. The Shapovalov form is the unique symmetric bilinear form

$$
M(m) \otimes M(m) \rightarrow \mathbb{C}
$$

characterized by the conditions

$$
S(v, v)=1 ; S(f x, y)=S(x, e y)
$$

for all $x, y \in M(m)$. One deduces easily from them that $S\left(f^{p} v, f^{q} v\right)=0$ for $p \neq q$, and $S\left(f^{p} v, f^{p} v\right)=c_{m, p}$, where

$$
c_{m, p}=p ! m(m-1) \cdots(m-p+1)
$$

We can consider $S$ as a map

$$
S: M \rightarrow M^{*}
$$

where $M^{*}=\oplus M_{p}^{*}, M_{p}^{*}$ being the dual space to $M^{p}$. The quotient $L(m)=M / \operatorname{ker} S$ is irreducible. If $m \notin \mathbb{N}$ then $S$ is an isomorphism. If $m \in \mathbb{N}$ then ker $S$ is the submodule generated by the singular vector $f^{m+1} v$ and we have an exact sequence

$$
0 \rightarrow M(-m-2) \rightarrow M(m) \rightarrow L(m) \rightarrow 0 .
$$

\subsection{Representations of $\tilde{\mathfrak{g}}$}

2.2.1. Let $\tilde{\mathfrak{g}}$ be the corresponding affine Lie algebra: $\tilde{\mathfrak{g}}=\mathfrak{g}\left[T, T^{-1}\right] \oplus \mathbb{C} c$ with the bracket

$$
\left[a T^{i}, b T^{j}\right]=[a, b] T^{2+j}+i(a, b) \delta_{i+j, 0} c
$$

$a, b \in \mathfrak{g}$ (see [K], ch. 7). Set $f_{0}=e T^{-1}, f_{1}=f, e_{0}=f T, e_{1}=e, h_{0}=c-h$, $h_{1}=h$. These elements generate $\tilde{\mathfrak{g}}$ and satisfy the usual relations corresponding to the Cartan matrix of type $A_{1}^{(1)},[\mathrm{K}]$. Throughout the paper we fix $k \in \mathbb{N}$ (the value of central charge). The action of $c$ on all representations of $\tilde{\mathfrak{g}}$ will be the multiplication by $k$.

2.2.2. Verma modules, Weyl modules and irreducible representations. For $m \in \mathbb{C}$ we denote by $M(k-m, m)$ the Verma module over $\tilde{\mathfrak{g}}$ generated by one vector $v$ subject to the defining relations $e_{i} v=0, i=1,2 ; h_{0} v=(k-m) v, h_{1} v=m v$.

Set $\mathfrak{g}^{+}=\mathfrak{g}[T] \subset \tilde{\mathfrak{g}}$; let $\epsilon: \mathfrak{g}^{+} \rightarrow \mathfrak{g}$ be the homomorphism sending $T$ to 0 . For a representation $M$ of $\mathfrak{g}$, consider the $\tilde{\mathfrak{g}}$-module induced from $\epsilon^{*} M$ by the imbedding $\tilde{\mathfrak{g}}^{+} \subset \tilde{\mathfrak{g}}$; denote by $\widehat{M}$ the quotient of this module by the relation $c=k . \widehat{M}$ is called the Weyl module corresponding to $M$. For example, $\widehat{M(m)}=M(k-m, m)$. For typographical reasons, we shall denote this module $\widehat{M}(m)$. 
If $m \in \mathbb{N}$, then $\widehat{M}(m)$ has singular vector $x_{1}=f_{1}^{m+1} v$, and we have an exact sequence

$$
\widehat{M}(-m-2) \rightarrow \widehat{M}(m) \rightarrow \widehat{L}(m) \rightarrow 0
$$

If in addition $m \leq k$ then $\widehat{M}(m)$ has another singular vector $x_{0}=f_{0}^{k-m+1} v$. We define $\bar{L}(m)$ to be the quotient of $\widehat{M}(m)$ over the submodule generated by $x_{0}$ and $x_{1}$. It is the unique irreducible quotient of $\widehat{M}(m)$. Such irreducibles are called integrable ([K], 10.4.6); they will be the most important for us.

We have an exact sequence

$$
\widehat{L}(2 k-m+2) \rightarrow \widehat{L}(m) \rightarrow \bar{L}(m) \rightarrow 0
$$

2.2.3. Set $\widetilde{f}_{0}=f T^{-1}, \widetilde{f}_{1}=e, \widetilde{e}_{0}=e T, \widetilde{e}_{1}=f, \widetilde{h}_{0}=h+c, \widetilde{h}_{1}=-h$. These elements give another set of the Chevalley generators of $\mathfrak{g}$.

Suppose that $m \in \mathbb{N}, m \leq k$. Let $\widetilde{M}(k-m, m)$ denote the Verma module generated by $\widetilde{v}$ subject to the relations $\widetilde{e}_{\imath} \widetilde{v}=0, \widetilde{h}_{0} \widetilde{v}=(k-m) \widetilde{v}, \widetilde{h}_{1} v=m \widetilde{v}$. Let $\overline{\widetilde{L}}(m)$ be its irreducible quotient. We have an isomorphism

$$
\overline{\widetilde{L}}(m) \cong L(\bar{m})
$$

sending $\widetilde{v}$ to $f^{m} v$.

\subsection{Spaces of Coinvariants}

Consider a complex projective line $\mathbb{P}^{1}$ with a fixed coordinate $z$, and $n+1$ distinct points $z_{1}, \ldots, z_{n}, z_{n+1}=\infty$ in it. Set $U=\mathbb{P}^{1}-\left\{z_{1}, \ldots, z_{n+1}\right\}$; let $\mathfrak{g}(U)$ be the Lie algebra of algebraic functions on $U$ with values in $\mathfrak{g}$.

At each point $z_{i}$ we have a local coordinate, namely $z-z_{i}$ for $0 \leq i \leq n$, and $1 / z$ for $i=n+1$. This defines the embedding

$$
\mathfrak{g}(U) \rightarrow \mathfrak{g}\left[\left[T, T^{-1}\right]\right]^{n+1},
$$

sending a function to a collection of its Laurent expansions at points $z_{1}, \ldots, z_{n+1}$.

Let $\widehat{\mathfrak{g}}^{n+1}$ denote the central extention of $\mathfrak{g}\left[\left[T, T^{-1}\right]\right]^{n+1}$ with the one-dimensional center, corresponding to the sum of cocycles (2.2.1). By the residue theorem, (7) lifts uniquely to the map

$$
\mathfrak{g}(U) \rightarrow \widehat{g}^{n+1},
$$

the lifting being defined by the same formula (7). Now, given representations $N_{1}, \ldots, N_{n+1}$ of $\tilde{\mathfrak{g}}$, their tensor product $N_{1} \otimes \cdots \otimes N_{n+1}$ is naturally a $\widehat{\mathfrak{g}}^{n+1}$ module, hence a $\mathfrak{g}(U)$-module, and we can form the space of coinvariants $\left(N_{1} \otimes \cdots \otimes N_{n+1}\right)_{\mathfrak{g}(U)}$.

2.3.1. Lemma. Suppose we are given $n+1 \mathfrak{g}$-modules $M_{1}, \ldots, M_{n+1}$. The canonical embeddings $M_{\imath} \rightarrow \widehat{M}_{i}$ induce the isomorphism

$$
\left(M_{1} \otimes \cdots \otimes M_{n+1}\right)_{\mathfrak{g}} \cong\left(\widehat{M}_{1} \otimes \cdots \otimes \widehat{M}_{n+1}\right)_{\mathfrak{g}(U)} .
$$


Proof. Since the embedding $M_{1} \otimes \cdots \otimes M_{n+1} \rightarrow \widehat{M}_{1} \otimes \cdots \otimes \widehat{M}_{n+1}$ is a homomorphism of $\mathfrak{g}$-modules, it induces the map

$$
\mathrm{U} \mathfrak{g}(U) \otimes_{\mathrm{Ug}}\left(M_{1} \otimes \cdots \otimes M_{n+1}\right) \rightarrow \widehat{M}_{1} \otimes \cdots \otimes \widehat{M}_{n+1} .
$$

( $\mathrm{Ug}$ denotes the enveloping algebra.)

Claim. The map (9) is an isomorphism.

Proof of the claim. Let us pick a $\mathbb{C}$-base $\left\{g_{1}, \ldots, g_{A}\right\}$ of $\mathfrak{g}$. This gives an ordered $\mathbb{C}$-base of $\tilde{\mathfrak{g}}$ :

$$
\left\{c ; g_{1} T^{i}, \ldots g_{A} T^{i}, i \in \mathbb{Z}\right\} \text {. }
$$

From the Poincaré-Birkhoff-Witt (PBW) theorem follows that for a $\widehat{\mathfrak{g}}$-module $M$, the module $\widehat{M}$ is a direct sum of subspaces

$$
\frac{g_{i_{1}}}{T^{j_{1}}} \ldots \frac{g_{i_{k}}}{T^{j_{k}}} M
$$

where the indices are: $0<j_{1} \leq j_{2} \leq \cdots \leq j_{k} ; 1 \leq i_{p} \leq A ; i_{p} \leq i_{p+1}$ if $j_{p}=j_{p+1}$.

On the other hand, it is not difficult to see that the functions $z^{i}, i \geq 0$, $\left(z-z_{p}\right)^{j}, j<0$, form a $\mathbb{C}$-base of the ring $\mathscr{O}(U)$ of algebraic functions on $U$. Analogously, this gives an evident basis of $\mathfrak{g}(U)$, and from PBW it follows that $\mathrm{Ug}(U) \otimes_{\mathrm{Ug}}\left(M_{1} \otimes \cdots \otimes M_{n+1}\right)$ is a direct sum of subspaces

$$
\begin{aligned}
& \frac{g_{i_{11}}}{\left(z-z_{1}\right)^{j_{11}}} \cdots \frac{g_{\imath_{1 k_{1}}}}{\left(z-z_{1}\right)^{j_{1 k_{1}}}} \frac{g_{i_{21}}}{\left(z-z_{2}\right)^{\jmath_{21}}} \cdots \frac{g_{\imath_{n k_{n}}}}{\left(z-z_{n}\right)^{j_{n k_{n}}}} \\
& \quad \times g_{\imath_{n+1,1}} z^{\jmath_{n+1,1}} \cdots g_{\imath_{n+1}, k_{n+1}} z^{\jmath_{n+1, k_{n+1}}} M_{1} \otimes \cdots \otimes M_{n+1},
\end{aligned}
$$

which map to the tensor product of subspaces (10) by the map (9). The claim follows from this.

Our lemma follows from it and from the Shapiro lemma.

2.3.2. Suppose we are given $n+1$ numbers $m_{1}, \ldots, m_{n+1} \in \mathbb{N}$ such that all $m_{\imath} \leq k$. The space of coinvariants

$$
W(z)=W\left(z_{1}, \ldots z_{n}\right)=\left(\bar{L}\left(m_{1}\right) \otimes \cdots \otimes \bar{L}\left(m_{n+1}\right)\right)_{\mathfrak{g}(U)}
$$

is called the space of conformal blocks (at a point $\left(z_{1}, \ldots, z_{n+1}\right)$ ). It is the main object of our study. When the point $\left(z_{1}, \ldots, z_{n}\right)$ varies, spaces $W(z)$ form a vector bundle $\mathscr{W}$ over $\mathbb{A}^{n}-\cup$ (diagonals). It is called the bundle of conformal blocks. The system (1) defines the flat connection in $\mathscr{W}$. In this Subsection we compute $W(z)$ in terms of the finite dimensional algebra $\mathfrak{g}$.

First, by Lemma 2.3.1 we have the isomorphism

$$
\left(\widehat{L}\left(m_{1}\right) \otimes \cdots \otimes \widehat{L}\left(m_{n+1}\right)\right)_{\mathfrak{g}(U)} \cong\left(L\left(m_{1}\right) \otimes \cdots \otimes L\left(m_{n+1}\right)\right)_{\mathfrak{g}} .
$$

set $L=L\left(m_{1}\right) \otimes \cdots \otimes L\left(m_{n}\right)$. The space $L$ is a sum of its $\mathfrak{h}$-homogenous components

$$
L=\sum_{l \in \mathbb{N}} L_{m-2 l},
$$

where we put $m=\sum_{i=1}^{n} m_{i}$. The operator $f$ acts as $f: L_{p} \rightarrow L_{p-2}$, and $e$ acts in the opposite direction. Set

$$
W_{p}=L_{p} / f L_{p+2}
$$


In other words, $W_{p}$ may be defined as

$$
W_{p}=L_{\mathfrak{n}_{-, p}} .
$$

Pick a lowest vector $v_{n+1}^{0} \in L\left(m_{n+1}\right)$, i.e. such a vector that $e v_{n+1}^{0}=0, v_{n+1}^{0} \neq 0$.

2.3.3. Lemma. The mapping

$$
x_{1} \otimes \cdots \otimes x_{n} \rightarrow x_{1} \otimes \cdots \otimes x_{n} \otimes v_{n+1}^{0}
$$

induces the isomorphism

$$
W_{m_{n+1}} \cong\left(L\left(m_{1}\right) \otimes \cdots \otimes L\left(m_{n+1}\right)\right)_{\mathfrak{g}} .
$$

Proof. Let us consider dual spaces. We have

$$
\left(\left(L\left(m_{1}\right) \otimes \cdots \otimes L\left(m_{n+1}\right)\right)_{\mathfrak{g}}\right)^{*} \cong \operatorname{Hom}_{\mathfrak{g}}\left(L\left(m_{n+1}\right), L^{*}\right)
$$

(in this proof $L^{*}$ denotes the dual representation, and not the contragradient one). The assignment $\phi \mapsto \phi\left(v_{n+1}^{0}\right)$ establishes the isomorphism of the last space with the space $\left\{x \in L_{-m_{n+1}}^{*} \mid f x=0\right\}$, and this space is evidently isomorphic to $W_{m_{n+1}}^{*}$.

2.3.4. The space $W_{m_{n+1}}$ may be non-zero only if the difference $m_{1}+\cdots+m_{n}-m_{n+1}$ is a nonnegative even integer. Set $N=\left(m_{1}+\cdots+m_{n}-m_{n+1}\right) / 2 ; s=k-m_{n+1}$. Let us consider the operator

$$
T_{s}(z)=\left(\sum_{i=1}^{n} z_{i} f^{(i)}\right)^{s+1}: W_{m_{n+1}+2(s+1)} \rightarrow W_{m_{n+1}},
$$

where $f^{(i)}: L \rightarrow L$ is the operator acting as $f$ on the $i^{\text {th }}$ factor $L\left(m_{i}\right)$ and as the identity on other factors. We will call $T_{s}(z)$ the truncation operator.

Set

$$
\bar{W}(z)=W_{m_{n+1}} / \operatorname{Im} T_{s}(z) .
$$

Here we set $W_{\imath}=0$ if $i>\sum_{i=1}^{n} m_{\imath}$, so $\bar{W}(z)=W_{m_{n+1}}$ if $m_{n+1}+2(s+1)>\sum_{i=1}^{n} m_{i}$. In other words, $T_{s}(z)$ is non-trivial only if

$$
s<N \text {. }
$$

This condition is equivalent to

$$
m_{1}+\cdots+m_{n+1}>2 k
$$

which is symmetric in $m_{\imath}$ (note that the sum is an even integer). We will call any of these equivalent conditions the truncation condition.

From (5) and 2.2.3 it follows that we have an exact sequence

$$
\widehat{L}\left(2 k-m_{n+1}+2\right) \rightarrow \widehat{L}\left(m_{n+1}\right) \rightarrow \bar{L}\left(m_{n+1}\right) \rightarrow 0 .
$$

Here the first arrow takes $v^{0} \in \widehat{L}\left(2 k-m_{n+1}+2\right)$ to $\left(f T^{-1}\right)^{s+1} v^{0} \in \widehat{L}\left(m_{n+1}\right)$, where we denoted by $v^{0} \in \widehat{L}(m)$ the vector $f^{m} v$. Let us apply to (15) the functor $\left(\widehat{L}\left(m_{1}\right) \otimes \cdots \otimes \widehat{L}\left(m_{n}\right) \otimes ?\right)_{\mathfrak{g}(U)}$. Using Lemma 2.3.1 and Lemma 2.3.3 we get an exact sequence

$$
W_{2 k-m_{n+1}+2} \rightarrow W_{m_{n+1}} \rightarrow\left(\widehat{L}\left(m_{1}\right) \otimes \cdots \otimes \widehat{L}\left(m_{n}\right) \otimes \bar{L}\left(m_{n+1}\right)\right)_{\mathfrak{g}(U)} \rightarrow 0 .
$$


Note that $2 k-m_{n+1}+2=m_{n+1}+2(s+1)$. The first arrow is identified with $T_{s}(z)$. Hence we get an isomorphism

$$
\bar{W}(z) \cong\left(\widehat{L}\left(m_{1}\right) \otimes \cdots \otimes \widehat{L}\left(m_{n}\right) \otimes \bar{L}\left(m_{n+1}\right)\right)_{\mathfrak{g}(U)} .
$$

It follows that we have an epimorphism

$$
\bar{W}(z) \rightarrow W(z) .
$$

2.3.5. Lemma. The map (17) is an isomorphism.

This result is due to A. Beilinson and the first author. The proof will appear elsewhere.

Summing up all the previous constructions, we get

2.3.6. Theorem. The mapping (11) induces the isomorphism $\bar{W}(z) \cong W(z)$.

\section{Sending to Differential Forms}

\subsection{Configurational Arrangements}

3.1.1. Let $X$ denote the $N$-fold product $\left(\mathbb{P}^{1}\right)^{N}$ with fixed coordinates $\left(t_{1}, \ldots, t_{N}\right)$, $t_{i} \in \mathbb{C} \cup\{\infty\}$. Pick $n$ distinct complex numbers $z_{1}, \ldots, z_{n}$; set $z_{n+1}=\infty$.

We shall consider the following set $\mathscr{C}$ of hyperplanes in $X$ :

$$
\begin{aligned}
\mathscr{C}= & \left\{H_{i j}: t_{\imath}=t_{j} ; i, j=1, \ldots, N ; i<j ;\right. \\
& \left.H_{i ; r}: t_{\imath}=z_{r}, i=1, \ldots, N ; r=1, \ldots, n+1\right\} .
\end{aligned}
$$

We set $U=X-\cup_{H \in \mathscr{E}} H$. We shall identify $X-\bigcup_{i=1}^{N} H_{\imath, n+1}$ with the $N$-dimensional affine space $\mathbb{A}^{N}$.

3.1.2. Twisted de Rham Complex. Pick complex numbers $m_{1}, \ldots, m_{n}$ and $\kappa \neq 0$. Let us assign to each hyperplane $H \in \mathscr{C}$ the number $a(H)$ as follows: set

for $r \neq n+1$,

$$
\begin{aligned}
& a\left(H_{i \jmath}\right)=a_{i j}=2 / \kappa, \\
& a\left(H_{i ; r}\right)=a_{i ; r}=-m_{i} / \kappa
\end{aligned}
$$

$$
a\left(H_{i ; n+1}\right)=a_{i ; n+1}=\left(m_{1}+\cdots+m_{n}-2(N-1)\right) / \kappa .
$$

The last numbers are determined from the requirement that for every line $L: t_{1}=$ $t_{2}=\cdots=t_{i}=t_{i+2}=\cdots=t_{N}=z_{r}$ the sum of the numbers $a(H)$ over all $H$ meeting $L$ transversally equals 0 .

Let us consider the following complex of $\mathbb{C}$-vector spaces $\Omega$. By definition, $\Omega^{i}$ is the space of complex holomorphic $i$-forms on $U$; set

$$
d=d_{\mathrm{DR}}+\alpha: \Omega^{2} \rightarrow \Omega^{i+1},
$$

where $d_{\mathrm{DR}}$ is the usual de Rham differential, and $\alpha$ is the left exterior multiplication by the form

$$
\alpha=\sum_{i, r: r \neq n+1} a_{i ; r} \mathrm{~d} \log \left(t_{\imath}-z_{r}\right)+\sum_{i<j} a_{\imath \jmath} \mathrm{d} \log \left(t_{\imath}-t_{\jmath}\right) .
$$


We shall write elements of $\Omega^{i}$ in the form $l \omega$, where $\omega$ is a holomorphic $i$-form on $U$, and

$$
l=\prod_{i, r}\left(t_{i}-z_{r}\right)^{a_{i ; r}} \prod_{\imath<j}\left(t_{i}-t_{j}\right)^{a_{i j}},
$$

where one should consider $l$ simply as a formal symbol. The formal differentiation gives

$$
d(l \omega)=l d \omega+(d l) \omega=l d \omega+l \alpha \omega=l\left(d_{D R}+\alpha\right) \omega,
$$

since $\alpha=d l / l$. Hence the twisted differential (18) takes the form of the usual exterior differential.

The geometrical meaning of $\Omega$ is as follows. The form $\alpha$ defines the integrable connection $\nabla=d_{\mathrm{DR}}+\alpha$ on the sheaf $\mathscr{Q}_{U}$ of holomorphic functions on $U . \Omega$ is the complex of global sections of the de Rham complex of $\left(\mathscr{O}_{U}, \nabla\right)$. If we denote by $\mathscr{S}$ the locally constant sheaf of horizontal sections, the cohomology $H^{\cdot}(U, \mathscr{S})$ is equal to $H^{\cdot}(\Omega)$. When the points $z_{1}, \ldots, z_{N}$ are moving these cohomology groups (and dual homology groups $H\left(U, \mathscr{S}^{*}\right)$ ) form a vector bundle with a flat connection (the Gauss-Manin connection). It is clear that the symmetric group $\sum_{N}$ acts on $X$ and on $\Omega$ by permutations of coordinates $t_{i}$.

\subsection{Resonances at Infinity}

From now on we pick the numerical data and adopt the notations of 2.3. Namely, we pick a positive integer $k$; integers $m_{\imath}, 0 \leq m_{\imath} \leq k ; i=1, \ldots, n+1$. We set $\kappa=k+2$ and $m=m_{1}+\cdots+m_{n}$. We suppose that $m-m_{n+1}$ is an even nonnegative integer, and set $\left.N=\left(m-m_{n+1}\right)\right) / 2$. We suppose that $m_{1}+\cdots+m_{n+1}>2 k$. Set $s=k-m_{n+1}$. We have $0 \leq s<N$ (cf. 2.3.4).

The rest of this subsection will not be needed in the sequel, although it sheds some light onto the reason for the appearance of new algebraic equations on our differential forms.

If $L$ is any intersection of hyperplanes from $\mathscr{C}$, we set

$$
a(L)=\sum_{H \supset L} a(H) .
$$

For a set of distinct $i_{1}, \ldots, i_{p}, 1 \leq i_{j} \leq N$ set

$$
L_{i_{1} \ldots i_{p}}=H_{\imath_{1} ; n+1} \cap \ldots \cap H_{\imath_{p} ; n+1} .
$$

We have

$$
a\left(L_{i_{1} \ldots i_{p}}\right)=\frac{p(m-2(N-1))}{\kappa}+\frac{p(p-1)}{2} \frac{2}{\kappa}=\frac{p}{\kappa}(k-s+p+1),
$$

since $m=k+2 N-s$. It follows that for $p=s+1$,

$$
a\left(L_{i_{1} \ldots i_{s+1}}\right)=s+1 .
$$

It is a positive integer, and we say that these edges $L_{i_{1} \ldots i_{s+1}}$ have "resonances." 


\subsection{The Map to Differential Forms}

3.3.1. Suppose we are given a sequence of integers $\left(i_{1}, \ldots, i_{q}\right), 0 \leq q \leq N$ such that for every $j 1 \leq i_{\jmath} \leq n$. Set

$$
\eta_{\left(i_{1}, \ldots, \imath_{q}\right)}=\frac{l d t_{1} \wedge \cdots \wedge d t_{q}}{\left(t_{1}-z_{\imath_{1}}\right) \cdots\left(t_{q}-z_{\imath_{q}}\right)} \in \Omega^{q} .
$$

Set

$$
\eta_{i_{1} \ldots i_{q}}=\sum_{\sigma \in \sum_{N}}(-1)^{|\sigma|} \sigma\left(\eta_{\left(\imath_{1}, \ldots, i_{q}\right)}\right) \in \Omega^{q},
$$

where the symmetric group acts by permutations of coordinates. Forms $\eta_{i_{1}, \ldots, i_{q}}$ do not depend on the order of indices.

Let us define the map

$$
\eta^{q}:\left(M\left(m_{1}\right) \otimes \cdots M\left(m_{n}\right)\right)_{m-2 q} \rightarrow \Omega^{q}
$$

as follows. Suppose we are given nonnegative integers $p_{1}, \ldots, p_{n} ; \sum p_{i}=q$. Choose any sequence $i_{1}, \ldots, i_{q}$ of integers such that for all $j, 1 \leq i_{j} \leq n$ and such that among $i_{j}$ 's there are exactly $p_{1}$ of 1 's, $p_{2}$ of 2's, $\ldots, p_{n}$ of $n$ 's. We shall write the last condition symbolically as

$$
\left\{i_{1}, \ldots, i_{q}\right\}=\left\{1^{p_{1}}, \ldots, n^{p_{n}}\right\} .
$$

Set

$$
\eta^{q}\left(f^{p_{1}} v_{1} \otimes \cdots \otimes f^{p_{n}} v_{n}\right)=\eta_{i_{1} \ldots i_{q}}
$$

Set

$$
\omega_{\imath_{1} \ldots \imath_{q}}=\left(c_{m_{1}, p_{1}} c_{m_{2}, p_{2}} \ldots c_{m_{n}, p_{n}} / \kappa^{q}\right) \eta_{i_{1} \ldots i_{q}}
$$

and

$$
\omega^{q}\left(f^{p_{1}} v_{1} \otimes \cdots f^{p_{n}} v_{n}\right)=\omega_{i_{1} \ldots i_{q}}
$$

where constants $c_{m, p}$ are as in (3).

From 2.1 it follows that $\omega^{q}$ induces maps

$$
\omega^{q}:\left(L\left(m_{1}\right) \otimes \cdots L\left(m_{n}\right)\right)_{m-2 q} \rightarrow \Omega^{q} .
$$

3.3.2. Lemma. (i) $\eta^{q}=0$ for $q<N-1$.

(ii) For any $x \in\left(L\left(m_{1}\right) \otimes \cdots L\left(m_{n}\right)\right)_{m-2(N-1)}$ we have

$$
\omega^{N}(f x)= \pm d \omega^{N-1}(x) .
$$

Proof. Direct calculation, or see [SV, 5.13, 6.6].

3.3.3. Corollary. The map $\omega^{N}$ induces the map

$$
\omega: W_{m_{n+1}} \rightarrow H^{N}(\Omega)
$$




\subsection{Truncation}

Let us consider the truncation operator

$$
T_{s}(z): L_{m_{n+1}+2(s+1)} \rightarrow L_{m_{n+1}}
$$

(cf. (12)), where we denote for brevity $L=L\left(m_{1}\right) \otimes \cdots \otimes L\left(m_{n}\right)$.

3.4.1. Main Theorem. The map $\omega^{N}(21)$ maps the image of $T_{s}(z)$ into $d \Omega^{N-1}$.

3.4.2. Corollary. The map $\omega^{N}$ induces the map

$$
\omega: W(z) \rightarrow H^{N}(\Omega) .
$$

Given a horizontal family of cycles $c(z) \in H_{N}\left(U, \mathscr{S}^{*}\right)$, the integration gives the horizontal section of the dual bundle of conformal blocks $\mathscr{W}^{*}$,

$$
\phi(z)=\int_{c(z)} \omega \in \mathscr{W}^{*}
$$

In other words, integral solutions of the $\mathrm{KZ}$ equations constructed in [SV] and given by the formulas from 3.3 lie in the bundle of conformal blocks.

3.4.3. Remark. In [V, (13), (14)] it is proven that maps (23) are injective.

\subsection{Proof of Theorem 3.4.1}

We shall use the following trick. Let us interchange in our projective arrangement the points $z_{1}$ and $z_{n+1}$. More precisely, let us make the change of variables: $u_{\imath}=\left(t_{i}-z_{1}\right)^{-1}, i=1, \ldots, N$. We have $t_{\imath}=u_{i}^{-1}+z_{1} ; d t_{\imath}=-u_{i}^{-2} d u_{i}$, hence

$$
t_{\imath}-z_{1}=u_{\imath}^{-1} ; t_{\imath}-z_{r}=-u_{i}^{-1} \widetilde{z}_{r}^{-1}\left(u_{i}-\widetilde{z}_{r}\right)
$$

for $j \neq i$, where $\widetilde{z}_{r}=\left(z_{r}-z_{1}\right)^{-1}$. Hence we have

$$
l= \pm A(\widetilde{z}) \prod_{i} u_{i}^{\left(m_{n+1}+2\right) / \kappa} \prod_{i ; r \geq 2}\left(u_{\imath}-\widetilde{z}_{r}\right)^{-m_{r} / \kappa} \prod_{i<j}\left(u_{\imath}-u_{\jmath}\right)^{2 / \kappa},
$$

where $A(\widetilde{z})=\prod_{r>2} \widetilde{z}_{r}^{N m_{r} / \kappa}$. Hence

$$
\begin{aligned}
l d t_{1} \wedge \cdots \wedge d t_{N}= & \pm A(\widetilde{z}) \prod_{i} u_{i}^{\left(m_{n+1}+2\right) / \kappa-2} \prod_{i ; r \geq 2}\left(u_{\imath}-\widetilde{z}_{r}\right)^{-m_{r} / \kappa} \\
& \prod_{\imath<j}\left(u_{i}-u_{j}\right)^{2 / \kappa} d u_{1} \wedge \cdots \wedge d u_{N}
\end{aligned}
$$

Let us set

$$
l^{\prime}(u)=\prod_{i} u^{\left(m_{n+1}+2\right) / \kappa-1} \prod_{i ; r \geq 2}\left(u_{i}-\widetilde{z}_{r}\right)^{-m_{r} / \kappa} \prod_{\imath<j}\left(u_{i}-u_{\jmath}\right)^{2 / \kappa} .
$$

Now let us rewrite forms $\eta, \omega$ in terms of new variables. Pick $i_{1}, \ldots, i_{N}$ such that $\left\{i_{1}, \ldots, i_{N}\right\}=\left\{1^{p_{1}}, \ldots, n^{p_{n}}\right\}$. We have

$$
\frac{1}{t_{i}-z_{r}}=-\frac{\widetilde{z}_{r} u_{i}}{u_{\imath}-\widetilde{z}_{r}}
$$


Suppose that $p_{1}=0$, i.e. all $i_{j}>1$. Then we have

$$
\eta_{\imath_{1} \ldots i_{N}}=\sum_{\sigma \in \sum_{N}} \sigma\left[ \pm A(\widetilde{z}) \prod_{j=1}^{N} \frac{-\widetilde{z}_{\imath_{j}}}{u_{j}-\widetilde{z}_{\imath_{j}}}\right] l^{\prime} d u= \pm A(\widetilde{z}) \prod_{j}\left(z_{1}-z_{i_{\jmath}}\right)^{-1} \eta_{i_{1} \ldots i_{N}}^{\prime}
$$

where $\eta^{\prime}$ denotes the form $\eta$ corresponding to $l^{\prime}$. Since

$$
\omega_{\imath_{1} \ldots i_{N}}=\operatorname{Const}\left(i_{1}, \ldots, i_{N}\right) \eta_{i_{1}, \ldots, \imath_{N}}
$$

it follows that

$$
\omega_{i_{1} \ldots i_{N}}= \pm A(\widetilde{z}) \prod_{\jmath=1}^{N}\left(z_{1}-z_{i_{\jmath}}\right)^{-1} \omega_{i_{1} \ldots i_{N}}^{\prime}
$$

where $i_{\jmath}>1$ for all $j$ and the sign \pm is the same for all $\left(i_{1}, \ldots, i_{N}\right)$.

Now recall that $m_{n+1}=k-s, 0 \leq s \leq N-1$, i.e. $\left(m_{n+1}+2\right) / \kappa=1-s / \kappa$. It follows that

$$
l^{\prime} d u=\prod_{\imath} u_{i}^{-s / \kappa} \prod_{i ; r \geq 2}\left(u_{\imath}-\widetilde{z}_{r}\right)^{-m_{\imath} / \kappa} \prod\left(u_{i}-u_{\jmath}\right)^{2 / \kappa} d u .
$$

In other words, the function $l^{\prime}$ corresponds to the situation when the representation $L(s)$ lives at the point 0 , and $L\left(m_{r}\right)$, for $r \geq 2$ live at the points $\widetilde{z}_{r}$. It follows that

$$
\omega_{1 \ldots 1 i_{s+2} \ldots i_{N}}^{\prime}=0
$$

for all $i_{s+2}, \ldots, i_{N}$. On the other hand, by Lemma 3.3 .2 (ii) for any $\left(i_{1}, \ldots, i_{N-1}\right)$ we have

$$
\sum_{i=1}^{n} \bar{\omega}_{i, i_{1}, \ldots, i_{N-1}}^{\prime}=0
$$

where $\bar{\omega}^{\prime}$ denotes the class modulo exact forms. Let us fix $i_{s+2}, \ldots, i_{N} \geq 2$. We have

$$
\begin{aligned}
0 & =\bar{\omega}_{1 \ldots i_{s+2}, \ldots, \imath_{N}}^{\prime}= \pm \sum_{{ }_{i_{1}, \ldots i_{s+1} \geq 2}} \bar{\omega}_{i_{1}, \ldots, l_{N}}^{\prime} \\
& = \pm A(\widetilde{z})^{-1} \sum_{i_{1}, \ldots i_{s+1} \geq 2}\left(z_{1}-z_{i_{1}}\right) \ldots\left(z_{1}-z_{i_{N}}\right) \bar{\omega}_{i_{1}, \ldots, i_{N}} \\
& =B(z) \sum_{i_{1}, \ldots, i_{s+1} \geq 2}\left(z_{1}-z_{\imath_{1}}\right) \ldots\left(z_{1}-z_{\imath_{s+1}}\right) \bar{\omega}_{\imath_{1}, \ldots, i_{N}} .
\end{aligned}
$$

Hence,

$$
\sum_{\imath_{1}, \ldots, \imath_{s+1} \geq 2}\left(z_{1}-z_{\imath_{1}}\right) \ldots\left(z_{1}-z_{i_{s+1}}\right) \bar{\omega}_{i_{1}, \ldots, \imath_{N}}=0
$$

Since

$$
\sum_{i=1}^{n} \bar{\omega}_{i, i_{1}, \ldots, i_{N-1}}=0
$$


we deduce that (27) holds true for any $i_{s+2}, \ldots, i_{N}$ (there might be indices equal to 1 among them). Again, from (28) follows that

$$
\sum_{\jmath \geq 2}\left(z_{1}-z_{j}\right) \bar{\omega}_{j i_{2} \ldots i_{N}}=-\sum_{\jmath \geq 1} z_{j} \bar{\omega}_{j i_{2} \ldots i_{N}}
$$

So, we can rewrite (27) in the form

$$
\sum_{j_{1}, \ldots, j_{s+1}} z_{\jmath_{1}} \ldots z_{j_{s+1}} \bar{\omega}_{j_{1} \ldots j_{s+1} i_{s+2} \ldots i_{N}}=0
$$

which is equivalent to saying that

$\omega\left(T_{s}(z) y\right)$ is an exact form for every $y \in L_{m_{n+1}+2(s+1)}$. QED.

\section{References}

[FSV] Feigin, B., Schechtman, V., Varchenko, A.: On algebraic equations satisfied by correlators in Wess-Zumino-Witten models. Lett. Math. Phys. 20, 291-297 (1990)

[K] Kac, V.: Infinite dimensional Lie algebras. Cambridge: Cambridge Univ. Press, 1985

[KZ] Knizhnik, V., Zamolodchikov, A.: Current algebra and Wess-Zumino model in two dimensions. Nucl. Phys. B247, 83 (1984)

[SV] Schechtman, V., Varchenko, A.: Arrangements of hyperplanes and Lie algebra homology. Invent. Math. 106, 134-194 (1991)

[TK] Tsuchia, A., Kanie, V.: Vertex operators in conformal field theory on $\mathbb{P}^{1}$ and monodromy representations of braid groups. Adv. Stud. Pure Math. 16, 297-372 (1988)

[V] Varchenko, A.: The function $\prod_{\imath<j}\left(t_{\imath}-t_{\jmath}\right)^{a_{i j} / \kappa}$ and the representation theory of Lie algebras and quantum groups. Preprint, 1992

Communicated by N.Yu. Reshetikhin 